\title{
A new SOGI-PLL method based on fuzzy logic for grid connected PV inverter
}

\author{
Abdelhadi Bouknadel, Naima Ikken, Ahmed Haddou, Nour-Eddine Tariba, \\ Hafsa El Omari, Hamid El Omari \\ Laboratory of Renewable Energy, Environment and Development (LERED), Faculty of Science and Technology, \\ University Hassan 1st, Morocco
}

\section{Article Info}

Article history:

Received May 30, 2018

Revised Jan 21, 2019

Accepted Mar 4, 2019

\section{Keywords:}

Fuzzy logic controller Grid-connected inverter Phase-locked loop (PLL) Second-order generalized integrator (SOGI)

\begin{abstract}
Phase angle detection of the grid voltage is an imperative part of control in most applications, especially for the synchronization of the current injected by the grid-connected photovoltaic inverters. Consequently, fast and accurate detection of the phase angle, frequency and amplitude of the grid voltage are indispensable data to ensure a correct generation of reference signals and operation of the grid connected inverters. We present in this work a new phase-locked loop (PLL) method for single-phase systems. The novelty is to generate an orthogonal voltage system using a second-order generalized integrator (SOGI), followed by a Park transformation, whose quadrature component is forced to zero by the fuzzy logic, in order to obtain rapid detection and a more accurate picture of the phase angle. Furthermore, simulation results with PSIM software will be submitted to verify the performance and effectiveness of the proposed method strategy. Finally, the experimental test will be used to extract the result and discuss the validity of the proposed algorithm.
\end{abstract}

Copyright (ㅇ 2019 Institute of Advanced Engineering and Science. All rights reserved.

\section{Corresponding Author:}

Abdelhadi Bouknadel,

Laboratory of Renewable Energy, Environment and Development (LERED),

Faculty of Science and Technology,

University Hassan 1st,

FSTS, Km7, Road Casablanca, Settat, Morocco.

Email: abdelhadi.bouknadel@gmail.com

\section{INTRODUCTION}

In the last decades, energy demand has been increasing due to lifestyle changes; households are now using more and more electronics and smart devices. Unfortunately in contrast to the upraised demand, conventional fossil fuels are decreasing, therefore to meet consumer needs, science researchers and industry leaders have made the utilization of renewable energy sources as an alternative solution to preserve power energy, mainly photovoltaic energy source.

Renewable energy sources have been used for more than 20 years, but there are some points where improvements are needed to increase the efficiency and quality of the energy injected into the grid. In fact, one of the most important problems is the synchronization of the injected current with the mains voltage, a better synchronization allows the extraction of the real phase angle for the different disturbances of the grid, in order to control the delay between the injected current and the mains voltage, and thus control the active and reactive power for a better performance [1-2].

For the latter case, among the problems that must be solved with a great caution we can mention the problem of the synchronization. In fact, the synchronization is used to implement a strategy to apply electronic control of the interconnection of this type of energy sources to the electrical network. a better synchronization allows the extraction of the real phase angle for the different disturbances of the grid, 
in order to control the delay between the injected current and the mains voltage, and thus control the active and reactive power for a better performance. Its purpose is to support the power system in case of a possibledefault, and take account various disturbances that can occur on the electrical network (change of amplitude, phase jump, harmonics, imbalance).It is generally, based on rapid and accurate detection of the phase angle of the grid voltage of electrical network, which can be estimated with the aid of a phaselocked loop (PLL) [3-4]. Most popular synchronization approaches for grid connected inverter with the grid voltage are based on the phase-locked loops (PLL) algorithms as shown in Figure 1 [4-6].

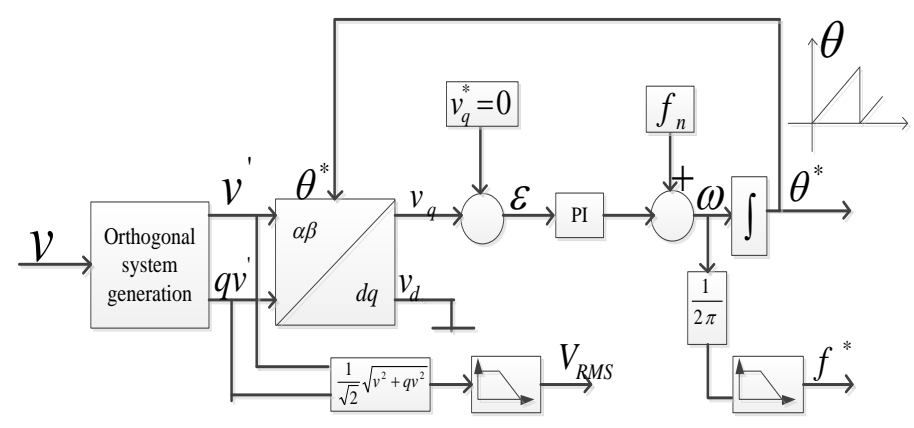

Figure 1. General structure of the phase-locked loop (PLL)

PLL design should have a rapid release and a very low inaccuracy between the obtained phase angle and the real phase angle, mainly at amplitude or frequency variation [6]-[12]. These two objectives cannot be achieved simultaneously. Either they get a fast response with medium precision or good accuracy with a longer synchronization time. Most PLL systems are implemented with a PI controller that is very sensitive to disturbances, as the controller parameters are synthesized around a specific operating point. The key drawback of this corrector (PI) is its inability to react to sudden changes of the grid voltage. The control device shall be optimized in order to achieve rapid locking with acceptable accuracy for different operating points.

For easier implementation researchers utilized various types of proportional integral (PI) controllers to control the PLL. However, the well-known disadvantages of the conventional PI and their adaptive versions encourage researchers to apply the intelligent controllers such fuzzy logic, neural network, neurofuzzy, etc. Among the main advantages of intelligent controllers for grid connected inverter system [6-11] are: their ability to deal with any non-linearity of arbitrary complexity and their designs do not require a mathematical model of the system and theoretically. Thus, the intelligent controllers are ideally suited for controlling grid connected inverter system. Among the various intelligent controllers fuzzy logic controller (FLC) is the simplest control and better than the conventional controllers in terms of insensitivity to parameter and grid variations, response time, settling time and robustness [13-15].

This document presents a new PLL method for grid-connected PV inverter. The new method consists in generating an orthogonal axis in a rotating frame, using a structure based on the integration of the generalized second order (SOGI), followed by a Park transformation to bring it back to a fixed reference [3]. The locking of the phase angle is translated by the cancellation of the quadratic component of the Park transform, using fuzzy logic. To validate the performances of this new method, we will proceed initially by a simulation under the PSIM software, by using the DLL block for the implementation of the proposed algorithm, and see its response against grid disturbances. After, we will experiment the algorithm, on a real conditions using a prototype based on a microcontroller STM32F407. For validation, we will visualize the shifted grid voltage sent to the ADC block with the voltage generated by the DAC block that represents a sinusoid of the estimated phase angle.

\section{PLL BASED ON THE SECOND ORDER GENERALIZED INTEGRATOR (SOGI-PLL)}

The structure of the second order generalized integrator (SOGI) is illustrated in Figure 2. The input signal v' is the voltage signal measured at the PCC. As output signals, two sinusoidal waves v'and qv' with a phase shift of $\frac{\pi}{2}$ are generated so that the component has the same phase and amplitude as the fundamental input voltage signal (v) [16-18]. The Figure 2, shows the structure of the second order generalized integrator (SOGI). 


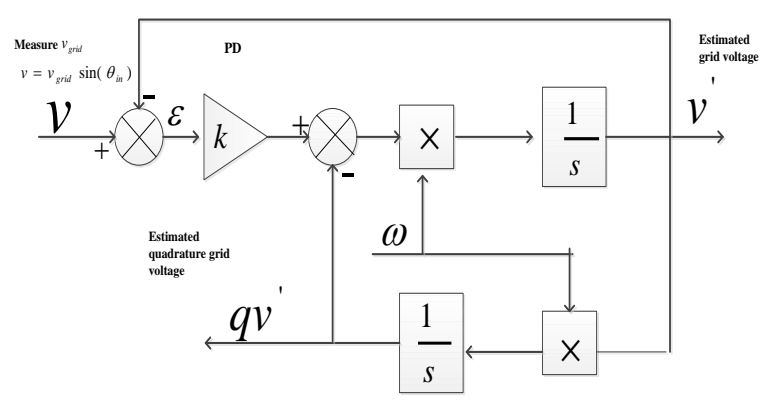

Figure 2. Orthogonal signal generator based on SOGI

The second-order generalized integrator (SOGI) acts as a band pass filter with infinite gain whose transfer function is defined in (1). The closed loop transfer functions and of the structure shown in Figure 2 are defined as [19-22]:

$$
\begin{aligned}
& H_{S O G I}=\frac{\omega_{n} s}{s^{2}+\omega_{n}^{2}} \\
& H_{d}(s)=\frac{v^{\prime}}{v}(s)=\frac{k \omega_{n} s}{s^{2}+k \omega_{n} s+\omega_{n}^{2}} \\
& H_{q}(s)=\frac{q v^{\prime}}{v}(s)=\frac{k \omega_{n}^{2}}{s^{2}+k \omega_{n} s+\omega_{n}^{2}}
\end{aligned}
$$

where $\omega_{n}$ represents the unamortized natural frequency of the SOGI that is equal to the estimated frequency $\omega_{n}=\omega$ and $k$ is the gain that affects the SOGI bandwidth.

The grid frequency can change; therefore, this orthogonal signal generator must be able to tune its coefficients in case of grid frequency change. To achieve this, trapezoidal approximation is used to get the discrete transfer function as follows:

$$
H_{d}(z)=\frac{k \omega_{n} \frac{2 z-1}{T_{S} z+1}}{\left(\frac{2 z-1}{T_{S} z+1}\right)^{2}+k \omega_{n} \frac{2 z-1}{T_{S} z+1}+\omega_{n}^{2}}=\frac{\left(2 k \omega_{n} T_{S}\right)\left(z^{2}-1\right)}{4(z-1)^{2}+\left(2 k \omega_{n} T_{S}\right)\left(z^{2}-1\right)+\left(\omega_{n} T_{S}\right)^{2}\left(z^{2}-1\right)^{2}}
$$

Now, using $x=2 k \omega_{n} T_{s}$ and $y=\left(\omega_{n} T_{s}\right)^{2}$

$$
\begin{aligned}
& H_{d}(z)=\frac{\left(\frac{x}{x+y+4}\right)+2\left(\frac{-x}{x+y+4}\right)^{2}}{\left(\frac{2(4-y)}{x+y+4}\right) z^{-1}-\left(\frac{x-y-4}{x+y+4}\right) z^{-2}}=\frac{b_{0}+b_{2} z^{-2}}{1-a_{1} z^{-1}-a_{2} z^{-2}} \\
& H_{q}(z)=\frac{\left(\frac{k \cdot y}{x+y+4}\right)+2\left(\frac{k \cdot y}{x+y+4}\right) z^{-1}+\left(\frac{k \cdot y}{x+y+4}\right) z^{-2}}{\left(\frac{2(4-y)}{x+y+4}\right) z^{-1}-\left(\frac{x-y-4}{x+y+4}\right) z^{-2}}=\frac{q b_{0}+q b_{1} z^{-1}+q b_{2} z^{-2}}{1-a_{1} z^{-1}-a_{2} z^{-2}}
\end{aligned}
$$

Once the orthogonal signal has been generated, park transform is used to detect the Q and D components on the rotating reference frame. This is then fed to the PI loop that controls the VCO of the PLL. The coefficients of the orthogonal signal generator can be tuned for varying grid frequency and sampling time [20-22].

As can be seen, the transfer function for $\mathrm{H}_{\mathrm{d}}$ resembles that of a Band Pass Filter, that filters out harmonic and random noise and whose output is in phase with that of the input signal. The transfer function for $\mathrm{H}_{\mathrm{q}}$ is the same as that of a second order Low Pass Filter, that not only filters out harmonics and random noise, but also introduces a phase shift of $\pi / 2$ radians [20-22]. Figure 3 Shows the block diagram of the overall SOGI-PLL structure [21-23]. 


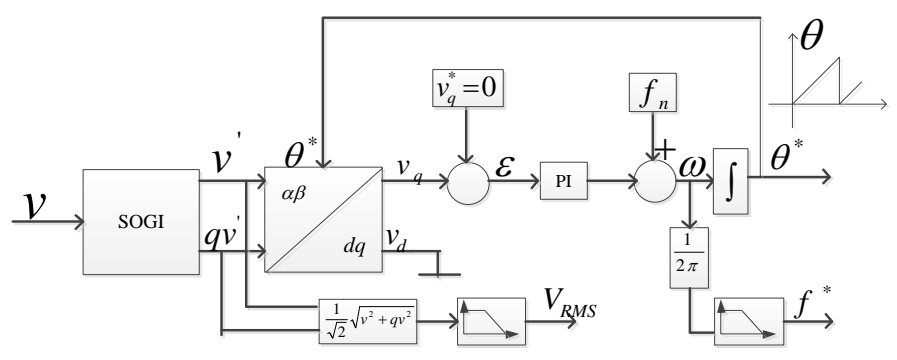

Figure 3. Structure of SOGI- PLL

\section{SOGI-PLL PROPOSED BASED ON FUZZY LOGIC}

This section presents a new phase-locked loop control (PLL) for a grid connected photovoltaic inverters. The originality in this work is to generate an orthogonal power voltage system based on a second order generalized integration structure (SOGI) driven by fuzzy logic to obtain fast detection and a more accurate image of the phase angle [24-25]. The Figure 4 shows the proposed second order generalized integrator (SOGI) structure based on fuzzy logic.

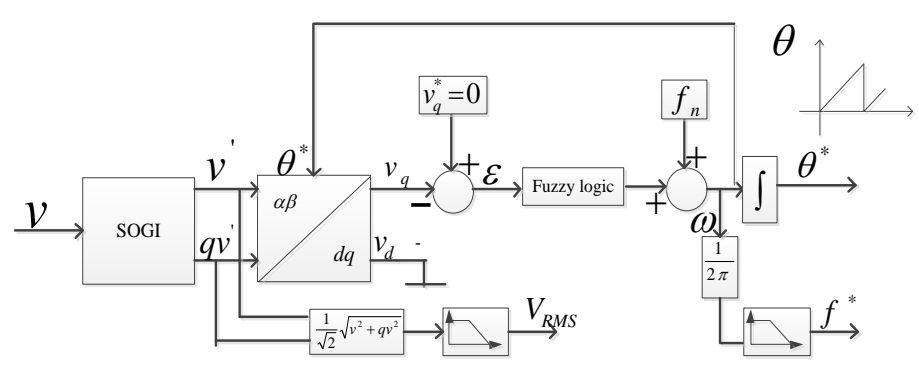

Figure 4. Proposed structure of SOGI-PLL based on fuzzy logic

The proposed method is based on the application of the fuzzy logic on the quadratic component of Park and its error compared to a reference value, to have a precise and fast correction of the frequency, which will be integrated to obtain the phase angle of the grid. For the validation of this method, we will perform the discretization and the implementation of the equations developed in the previous section, in the form of the functions in programming language C. Firstly, they will be used in the DLL block which executes the routines at each step simulation of the PSIM software. Secondly, they will be used for the generation of the program that will be embedded in the STM32F407 microcontroller for experimental validation.

\subsection{Fuzzy logic controller design}

Defining input and output variables, and controller functions is one of the most important steps in the fuzzy logic design process. In this study, the selection of members and the number of rules had to be minimized. Blurred affiliations and rules must act quickly to speed up or move backwards the d-q axis until it reaches synchronization speed and position, then lock the axis to its correct synchronization state. This requires more attention to the design of exit rules [1-2].

The fuzzy logic controller is designed with two variables (quadrature component of Park and its error to a reference value) defined as input variables. These two input variables have five triangle membership functions for each of them. The linguistic variables "positive large (PL)","positive small (PS)","zero (Z)","negative small (NS)","negative large (NL)" for two input variables are used to express the fuzzy variables. Thus the control action is defuzzified in a unit range also with five membership functions defined as output variable of fuzzy logic to express the values of frequency correction [1, 26-28]. The detailed design of the fuzzy membership functions is illustrated in Figure 5. Established on the basis of information about the system and how it works, depending on error variations for a rapid transient response. The fuzzy logic rule bases for output variables are shown in Table 1. 


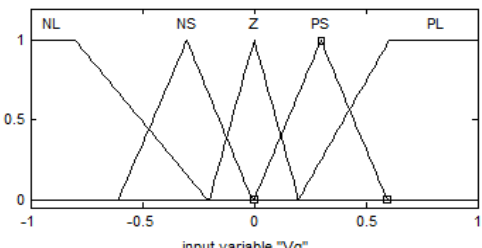

(a)

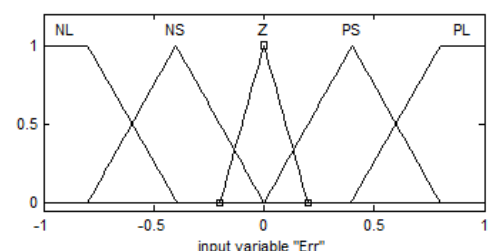

(b)

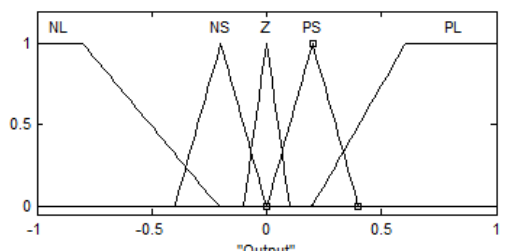

(c)

Figure 5. (a) Vq membership, (b) Error membership, (c) Output membership

Table 1. Basic rule for output variable

\begin{tabular}{cccccc}
\hline$E r r$ & & & $V r$ & \\
& $P L$ & $P S$ & $Z$ & $N Z$ & $N L$ \\
\hline$P L$ & $\mathrm{~L}$ & $\mathrm{~L}$ & $\mathrm{M}$ & $\mathrm{S}$ & $\mathrm{S}$ \\
$P S$ & $\mathrm{~L}$ & $\mathrm{M}$ & $\mathrm{M}$ & $\mathrm{M}$ & $\mathrm{S}$ \\
$Z$ & $\mathrm{M}$ & $\mathrm{M}$ & $\mathrm{M}$ & $\mathrm{M}$ & $\mathrm{M}$ \\
$N S$ & $\mathrm{~S}$ & $\mathrm{~S}$ & $\mathrm{M}$ & $\mathrm{L}$ & $\mathrm{L}$ \\
$N L$ & $\mathrm{~S}$ & $\mathrm{M}$ & $\mathrm{M}$ & $\mathrm{L}$ & $\mathrm{L}$ \\
\hline
\end{tabular}

\subsection{Discretization of the two methods}

a. Method SOGI with PI

/*Estimation of u value with the SOGI*/

$\operatorname{sogi} \_u[n]=(b 0 *(u[n]-u[n-2]))+\left(a 1 * \operatorname{sogi} \_u[n-1]\right)+\left(a 2 * \operatorname{sogi} \_u[n-2]\right) ;$

/*Update of $\mathrm{u} * /$

sogi_u[n-2]=sogi_u[n-1];

sogi_u[n-1]=sogi_u[n];

/*Estimation of $\mathrm{u}$ value with the SOGI */

sogi_qu[n] $=(\mathrm{qb} 0 * \mathrm{u}[\mathrm{n}])+(\mathrm{b} 1 * \mathrm{u}[\mathrm{n}-1])+(\mathrm{qb} 2 * \mathrm{u}[\mathrm{n}-2])+\left(\mathrm{a} 1 * \operatorname{sogi} \_\mathrm{qu}[\mathrm{n}-1]\right)+(\mathrm{a} 2 * \operatorname{sogi} \mathrm{qu}[\mathrm{n}-2])$

/*Update of qu*/

sogi_qu[n-2]=sogi_qu[n-1];sogi_qu[n-1]=sogi_qu[n];

$/ *$ update of grid voltage*/

$\mathrm{u}[\mathrm{n}-2]=\mathrm{u}[\mathrm{n}-1]$;

$\mathrm{u}[\mathrm{n}-1]=\mathrm{u}[\mathrm{n}]$;

/*Park Transform from alpha beta to d-q axis*/

u_Q[n] $=(\cos *$ sogi_u[n] $)+(\sin *$ sogi_qu[n] $)$;

u_D[n] $=(\cos *$ sogi_qu[n] $)$ - (sin*sogi_u[n] $)$;

/*PI process*/

/*Error update*/

Error $=\mathrm{u} \_\mathrm{Q}[\mathrm{n}]-\mathrm{u}$ _Qref;

$/ *$ proportional term */

up $=$ v.Kp*Error;

$/ *$ integral term */

$\mathrm{ui}=(\mathrm{v} . \mathrm{Ki} * \mathrm{up})+\mathrm{i} 1$;

i1 =ui;

/* sum of integral and proportional term */

PI_f[n] = up + ui;

$/ *$ Estimation of outputfrequency*/

fo=fn+PI_f[n];

/*Update of Uq value of Park component */

u_Q[n-1]=u_Q[n];

$/ *$ Frequency integration to obtain the phase angle*/

theta[n]=theta[n-1] $+(2.0 * \pi *$ timestep*fo $)$;

$/ *$ Re-initialization of phase angle over 2pi*/

if $($ theta $[\mathrm{n}]>(2 \pi))$

theta[n] $=0$;

/*Update of phase angle*/

Theta[n-1] $=$ theta[n]; 
b. Method SOGI with fuzzy logic

// Estimation of $u$ value with the SOGI //

sogi_u[n] $=(b 0 *(u[n]-u[n-2]))+\left(a 1 * \operatorname{sogi} \_u[n-1]\right)+(a 2 * \operatorname{sogi} u[[n-2])$;

// updat of $\mathrm{u} / /$

sogi_u[n-2]=sogi_u[n-1];

sogi_u[n-1]=sogi_u[n];

// Estimation of $u$ value with the SOGI //

sogi_qu[n] $=(\mathrm{qb} 0 * \mathrm{u}[\mathrm{n}])+(\mathrm{b} 1 * \mathrm{u}[\mathrm{n}-1])+(\mathrm{qb} 2 * \mathrm{u}[\mathrm{n}-2])+\left(\mathrm{a} 1 * \operatorname{sogi} \_\mathrm{qu}[\mathrm{n}-1]\right)+(\mathrm{a} 2 * \operatorname{sogi}$ qu$[\mathrm{n}-2])$

// update of qu //

sogi_qu[n-2]=sogi_qu[n-1];

sogi_qu[n-1]=sogi_qu[n];

// update of grid voltage //

$\mathrm{u}[\mathrm{n}-2]=\mathrm{u}[\mathrm{n}-1]$;

$\mathrm{u}[\mathrm{n}-1]=\mathrm{u}[\mathrm{n}]$;

// Park Transform from alpha beta to $\mathrm{d}-\mathrm{q}$ axis //

u_Q[n]=(cos*sogi_u[n] $)+(\sin *$ sogi_qu[n] $)$;

u_D[n]=(cos*sogi_qu[n] $)-(\sin * \operatorname{sogi} u[n])$;

// Fuzzyprocess//

// Error update //

Error=u_Q[n]-u_Qref;

// Fuzzification of the q axis input voltage//

Fuzzification(u_Q[0],X1,X1_abcs);

// Fuzzification of the q axis error//

Fuzzification(Error,X2,X2_abcs);

// Application of basic rules between uq and Error to have fuzzified the correction frequency//

FuzzyInference(X1, X2, Y);

//Defuzzification of the frequency correction //

Fuzzy_f[n]=Difuzzification(Y,OutputFunc);

// Application of the frequency correction//

fo=fnom+Fuzzy_f[n];

// Update of Uq value of Park component //

u_Q[n-1]=u_Q[n];

// Frequency integration to obtaine the phase angle //

theta[n]=theta $[n-1]+(2.0 * \pi *$ timestep $*$ fo $)$;

// Re-initialization of phase angle over 2pi //

if $($ theta $[\mathrm{n}]>(2 \pi))$

theta $[\mathrm{n}]=0$;

// Update of phase angle//

theta $[\mathrm{n}-1]=$ theta $[\mathrm{n}]$;

\subsection{Simulation results}

In this section, we present the simulation results under the PSIM software, the Figure 6, represents the two blocks used in the simulation, a $\mathrm{C}$ block that generates a sinusoidal signal controlled in frequency and amplitude to simulate the disturbances of the grid, and a DLL block that contains the functions SOGI, Park, fuzzy logic and VCO for the estimation of the phase angle of the grid.

The Figure 7 shows the results of the proposed method with different variations (amplitude variation, frequency and phase shift). Figure 7 shows that the proposed generalized integration structure (SOGI) of second order controlled by fuzzy logic, has a very good behavior against this variation despite transient oscillations (Amplitude variation as shown in Figure 7-a, Amplitude and frequency variation as shown in Figure 7-b, Frequency variation and phase jump as shown in Figure 7-c. Therefore, the response of the proposed method is very efficient and accurate, and this can be seen by comparing the generated sinusoidal signal with the estimated phase angle and the signal that simulates the grid, the locking time of the Phase angle is very fast (less than one period of the grid signal). 


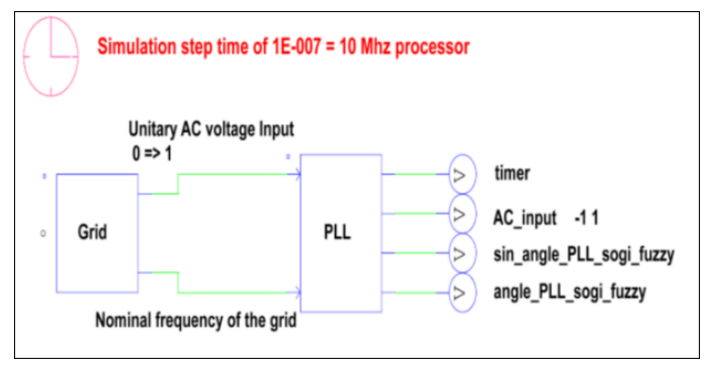

Figure 6. Simulation structure

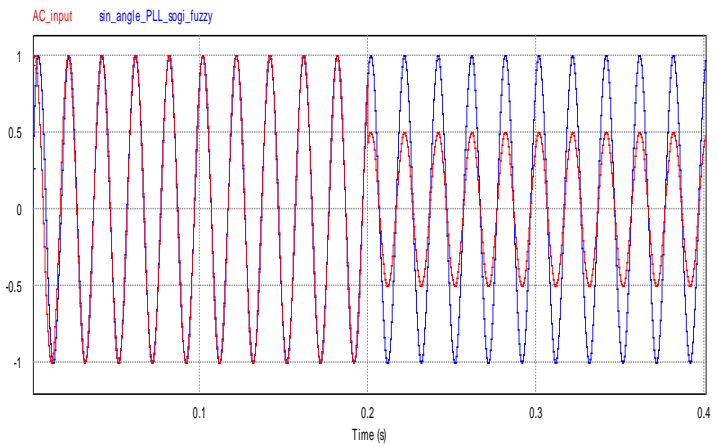

(a)

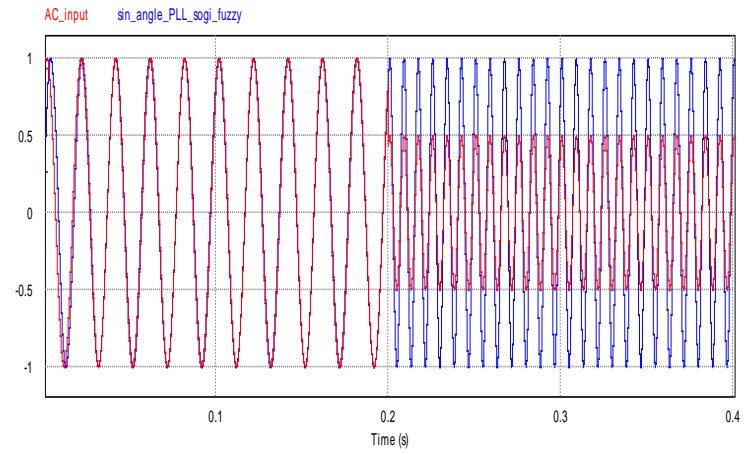

(b)

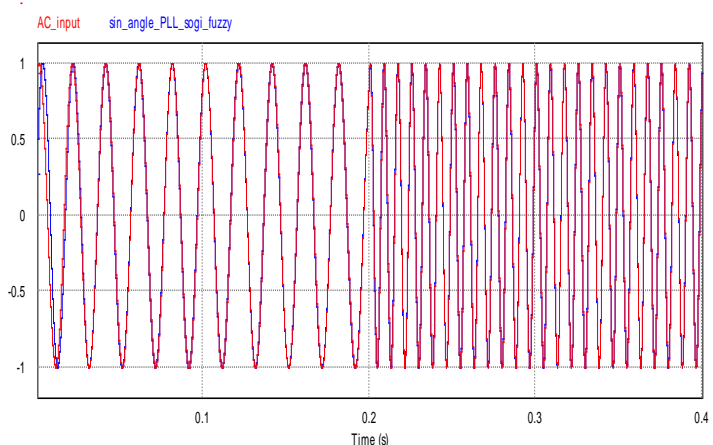

(c)

Figure 7. Results of the simulation, (a) Amplitude variation, (b) Amplitude and frequency variation, (c) Frequency variation and phase jump

\subsection{Experimental result}

The proposed new logic (SOGI-Fuzzy) is verified and evaluated by an experimental test, using a differential voltage level shift circuit to sample the network voltage with the CAN of the STM32F4discovery evaluation board, to evaluate the accuracy of SOGI-fuzzy logic, we visualize using a digital scoop the sinusoid of the estimated phase angle in the DAC with the offset gate voltage. The Figure 8 , shows the connection between the test equipment used in the experimental prototype. The proposed PLL structure based on second order generalized integrator is experimentally validated in the following. The Figure 9, shows the assembly of the practical test of the proposed method. 


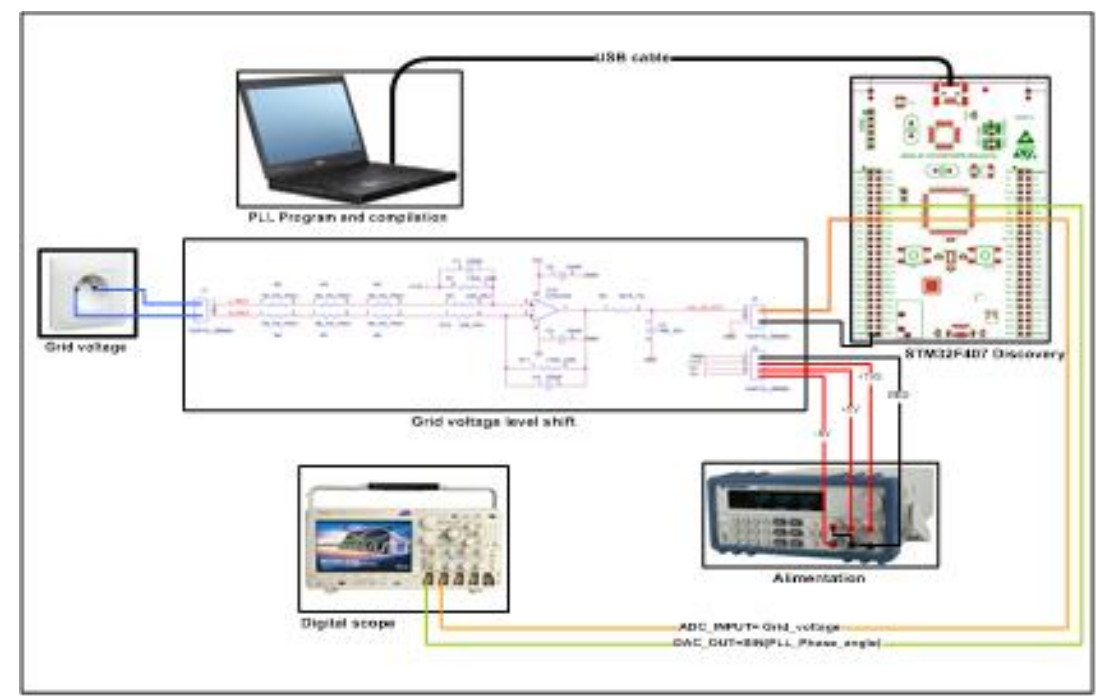

Figure 8. Connection of the experimental equipments

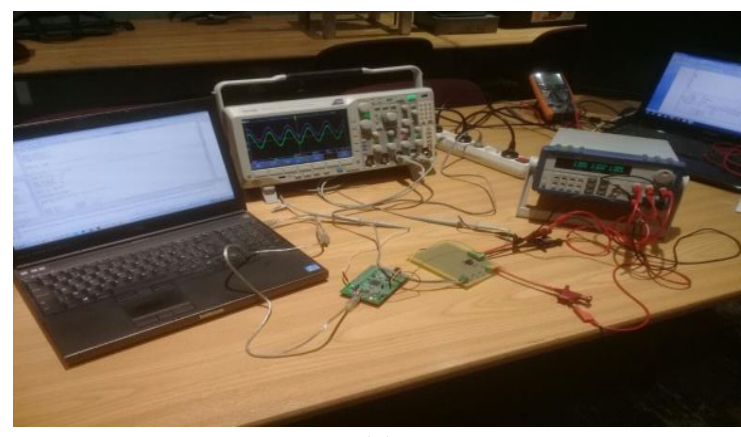

(a)

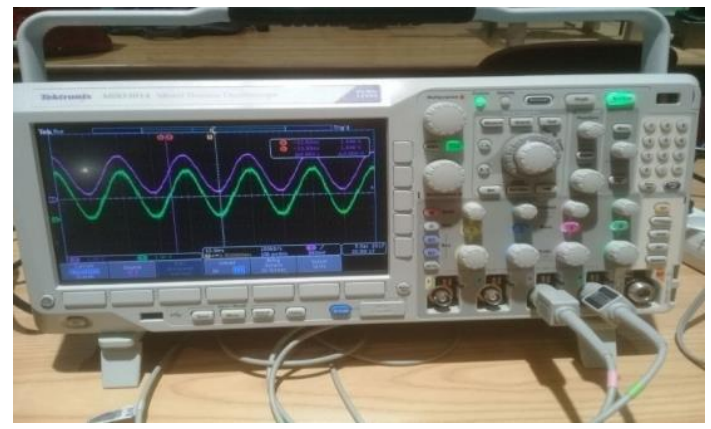

(b)

Figure 9. Results of the experimental tests, (a) Hardware platform connection, (b) Dynamic response of the inverter connected to the grid with the proposed method.

\subsection{Discussion}

Once the simulation task is complete, the process of reading the analog signal and generating the sinusoidal signal using the estimated phase angle is done with the STM32F407-discovery evaluation board. The functions of the SOGI-Fuzzy method runs in an interrupt with a frequency of 10khz, the complete program is compiled using the COOCOX software, that generates a Hex file which will be sent to the microcontroller of the evaluation board STM32F407-discovery.

The proposed PLL (SOGI-Fuzzy logic) is verified and evaluated by comprehensive tests. To evaluate the performance of the proposed method, different scenarios are carried out. In the first case, the stability of the SOGI-PLL must be simulated and experimentally tested for ideal grid conditions and not ideal with fuzzy logic control (the performance of the proposed method is studied both for stable operation and transient conditions such as reference variations). The second scenario, verifies the ability of SOGI-PLL to achieve fast and more accurate lock status. Thus the compensation gains are easily determined according to the system operating point from the PI controller control by the fuzzy logic. So in Figure 10, shows the sinusoidal signal generated by the DAC bloc (shifted by a voltage of $1.65 \mathrm{~V}$ ), with the grid voltage signal scaled by the differential voltage level shift circuit.

From the digital oscilloscope pictures, the results of the proposed SOGI-Fuzzy method have a fast response to transient's disturbance, and the output signal generated by the DAC tracks the network voltage with great accuracy. Thus, the simulation results are validated by experimental tests, and the new SOGIFuzzy method has demonstrated efficiency in terms of speed and accuracy. The SOGI-Fuzzy logic controller has also been designed not only to have a faster and more precise response, but also to have the ability to attenuate network disturbance (phase jump, amplitude change, harmonic, imbalance). 


\section{CONCLUSION}

In this article, we present a new method of synchronization based on the fuzzy logic for the correction of the estimated grid frequency, simulation and practical tests show that the new command gives a faster and more precise and effective response than the classical control based on the PI corrector. The new SOGI-FUZZY has also been designed not only to have a faster and more accurate response, but also to mitigate the disruption of the grid characteristics (phase jump, amplitude change, harmonic, imbalance).

\section{ACKNOWLEDGEMENTS}

The authors would like to thank IRESEN (Moroccan Research Institute for Solar and New Energy) for the financial support of the research project on $6 \mathrm{kVA}$ inverters.

\section{REFERENCES}

[1] Ikken N., Bouknadel A., Haddou A., et al. "A comparative study and implementation of single-phase PLL techniques for grid-connected inverters systems," Journal of Electrical Systems, vol. 14, no 4, 2018.

[2] Ikken N., Bouknadel A., El Omari Hafsa, et al. "Design and implementation of intelligent PI-fuzzy logic control for grid connected inverters," In: Renewable and Sustainable Energy Conference (IRSEC), 2016 International. IEEE, pp. 1111-1117, 2016.

[3] Jaalam N., Rahim N., Bakar A., et al., "A comprehensive review of synchronization methods for grid-connected converters of renewable energy source," Renew. Sustain. Energy Rev., vol. 59, pp. 1471-1481, 2016.

[4] Golestan S., Guerrero J.M., Vidal A., et al., "Small-signal modeling, stability analysis and design optimization of single-phase delay-based PLLs," IEEE Trans. Power Electron., vol. 31, no. 5, pp. 3517-3527, 2016.

[5] Barnes M, Siyu Gao, "Phase-locked loops for grid-tied inverters: comparison and testing," In Institution of Engineering and Technology, 2016 [cited 2018 May 1]. pp. 6.-6. Available from: http://digitallibrary.theiet.org/content/conferences/10.1049/cp.2016.0304.

[6] Xiao F, Dong L, Liao X. , "Fast reactive current detection method for single-phase grid-connected inverters," IET Power Electronics; vol. 9, no. 3, pp. 401-7, Mar 2016.

[7] Thumu R, Reddy KH, "A review on fuzzy-GA based controller for power flow control in grid connected pv system," International Journal of Electrical and Computer Engineering (IJECE), vol. 7, no. 1,pp. 125, Feb 2017.

[8] Bacon V.D. and S.A.O.D. Silva, "Performance improvement of a three-phase phase-locked-loop algorithm under utility voltage disturbances using non-autonomous adaptive filters, "IET Power Electronics, vol. 8, pp. 2237-2250, 2015.

[9] Lubura S., et al., "Single-phase phase locked loop with DC offset and noise rejection for photovoltaic inverters," IET Power Electronics, vol. 7, pp. 2288-2299, 2014.

[10] Elrayyah A., Y. Sozer and M. Elbuluk, "Robust phase locked-loop algorithm for single-phase utility-interactive inverters," IET Power Electronics, vol. 7, pp. 1064-1072, pp. 2014.

[11] Golestan S., Freijedo F.D., Guerrero J.M., "A systematic approach to design high-order phase-locked loops," IEEE Trans. Power Electron, vol. 30, no. 6, pp. 2885-2890, 2015.

[12] Serna J., "Synchrophasor measurement with polynomial phase-locked-loop taylor-fourier filters," IEEE Transactions on Instrumentation and Measurement, vol. 64, pp. 328-337, 2015.

[13] Yong Y, "Direct instantaneous power control of three-level grid-connected," International Journal of Electrical and Computer Engineering (IJECE), vol. 6, no. 3, pp. 1260, Jun 2016.

[14] Dongsul Shin, Kyoung-Jun Lee, Jong-Pil Lee, Dong-Wook Yoo, Hee-Je Kim, "Implementation of fault ridethrough techniques of grid-connected inverter for distributed energy resources with adaptive low-pass notch PLL," IEEE Transactions on Power Electronics, vol.30, no.5, pp. 2859-2871, May 2015.

[15] Ma C., Gao F., He G., et al., "A voltage detection method for the voltage ride-through operation of renewable energy generation systems under grid voltage distortion conditions," IEEE Trans. Sustain. Energy, vol. 6, no. 3, pp. 1131-1139, 2015.

[16] Siyu Gao and Mike Barnes, "Phase-locked loop for AC systems: analyses and comparisons," IET Power Eectronics Machines and Drives Conference, 2014.

[17] Lubura S., Šoja M., Lale S., et al. ,"Single-phase phase locked loop with DC offset and noise rejection for photovoltaic inverters," IET Power Electron, vol. 7, no. 9, pp. 2288-2299, 2014.

[18] Reza M. S., Ciobotaru M., Agelidis V. G., "Robust technique for accurate estimation of single-phase grid voltage fundamental frequency and amplitude," IET Gener. Transm. Distrib, vol. 9, no. 2, pp. 183-192, 2015.

[19] Bacon V. D., Silva S. A. O., Campanhol L. B. G., et al., "Stability analysis and performance evaluation of a singlephase phase-locked loop algorithm using a non-autonomous adaptive filter," IET Power Electron, vol. 7, no. 8, pp. 2081-2092, , 2014.

[20] Elrayyah A., Sozer Y., Elbuluk M., "Robust phase locked-loop algorithm for single-phase utility-interactive inverters', IET Power Electron, vol. 7, no. 5, pp. 1064-1072, 2014.

[21] Fuad Khaled, et al. "Grid-voltage Synchronization Algorithms Based on Phase-locked Loop and Frequency-locked Loop for Power Converters," Thesis submitted for examination for the degree of Master of Science in Technology, Aalto university, 2014. 
[22] Yang Yongheng, Blaabjerg Frede, Zou Zhixiang, "Benchmarking of grid fault modes in single-phase gridconnected photovoltaic systems," IEEE Transactions on Industry Applications, vol. 49, no 5, pp. 2167-2176, 2013.

[23] Rennane A., Saheb-koussa D., "Evaluation des performances de la boucle à verrouillage de phase (PLL) pour l'interconnexion d'une source d'énergie renouvelable au réseau électrique," Revue des Energies Renouvelables, vol. 17, no 2, pp. 227-243, 2014.

[24] Kjaer Soeren Baekhoej, Pedersen John K., Blaabjerg Frede, "A review of single-phase grid-connected inverters for photovoltaic modules," IEEE transactions on industry applications, vol. 41, no 5, pp. 1292-1306, 2005.

[25] Bhardwaj M. "Software phased-locked loop design using C2000 ${ }^{\mathrm{TM}}$ microcontrollers for single phase grid connected inverter," Application Report, Texas Instruments, 2013.

[26] Hamed Hany A., Bayoumi Ehab HE, et El-kholy E. E., "Fuzzy PLL for three-level neutral point clamped active rectifiers" International Journal of Industrial Electronics and Drives, vol. 2, no 3, pp. 170-190, 2015.

[27] "Digitally Controlled Solar Micro Inverter Design using C2000 Piccolo Microcontroller," Application Report, Texas Instruments, 2013.

[28] Ciobotaru Mihai, Teodorescu Remus, Blaabjerg Frede, et al. "A new single-phase PLL structure based on second order generalized integrator," In Power Electronics Specialists Conference, pp. 1-6, 2006. 